\section{INTRA-ARTERIAL THIOPENTONE REPORT OF A CASE}

BY

PETER STUART, M.B., F.F.A. R.C.S., D.A. Senior Registrar, Department of Anaesthetics, Glasgow Royal Infirmary

Thiopentone sodium (sodium ethylmethylbutyl thiobarbiturate) was synthesized by Volwiler and Tabern in 1932. Since its first introduction into clinical practice by Lundy in 1934, it has been used by the medical profession throughout the world, particularly as an intravenous agent in anaesthesia.

Cases of accidental intra-arterial injection have been reported in the literature from time to time, the position being well reviewed by Cohen (1948) in a study of 12 cases, 8 of them previously unpublished. As Sir Robert Macintosh has stated on more than one occasion, the repetition of these mistakes can be avoided only by reporting the details of any such misfortune.

\section{Case Report}

A healthy young man aged 19 was admitted for circumcision. Premedication consisted of papaveretum, 1/3 gr. (22 mg.), and scopolamine, 1/150 gr. (0.43 mg.).

At 10 a.m. on May 21, 1955, anaesthesia was induced with $5 \%$ thiopentone solution, injection being made into the left median cubital vein on the anterior aspect of the upper forearm. Injection of $1 \mathrm{ml}$. was followed by the usual pause, during which the patient was asked whether he felt any pain in the forearm or hand (a routine precaution); on receiving a negative reply a further $5 \mathrm{ml}$. was injected. At this point he complained of a burning sensation, rapidly intensifying, in his forearm and hand, the severity of which caused him to writhe in agony on the trolley, to an extent requiring forcible restraint. The onset of narcosis was significantly and obviously delayed, but apart from slight flushing of the hand, especially the fingers, there was no other visible abnormality at this stage. Anaesthesia was continued, using cyclopropane and oxygen on a closed circuit with carbon dioxide absorption, and a brachial plexus block was immediately performed, using lignocaine $2 \%(20 \mathrm{ml}$.) and $1 \%(10 \mathrm{ml}$.). On completion of the block $5 \mathrm{ml}$. of $1 \%$ lignocaine was injected into the subclavian artery. The surgeon was requested to ensure complete haemostasis in view of the forthcoming heparinization.

On return to the ward further treatment was carried out as follows: (1) Heparinization: 10,000 units intravenously and repeated six-hourly; (2) sedation: morphine, 1/6 gr. (11 mg.) six-hourly; (3) elevation of limb, which was swathed in cotton-wool.

On examination of the limb the success of the brachial plexus block was confirmed by the presence of both motor and sensory paralysis with satisfactory vasodilatation, the limb being warm and pink; both radial and ulnar pulses were present. At the site of injection a superficial ulnar artery was palpable, pulsation being visible on closer inspection; this vessel was palpable for approximately $2 \frac{1}{2}$ in. $(6.3 \mathrm{~cm}$.) into the forearm. A corresponding artery in the right forearm was palpable for six inches $(15 \mathrm{~cm}$.).

At 2.30 p.m. the brachial plexus block had worn off. The skin temperature of the left hand and forearm was much lower than that of the right, with pallor of the digits and an increased arteriolar capillary refill time. The ulnar pulse was barely palpable on the affected side. There was no evidence of oedema of the hand or forearm. The following measures were carried out: (1) Brachial plexus block was repeated, using $50 \mathrm{ml}$. of 1 in 1,500 cinchocaine ; paraesthesiae were elicited. (2) Intra-arterial tolazoline ("priscol "), $50 \mathrm{mg}$. in $1 \%$ solution, and heparin, 5,000 units, were injected into the subclavian artery. (3) Local infiltration of the left antecubital fossa and upper forearm along the course of the superficial ulnar artery, using $20 \mathrm{ml}$. of $0.5 \%$ lignocaine with 1,000 units of hyalase. The effect of this procedure was to produce again a very satisfactory vasodilatation with a warm pink limb, the condition of which remained unchanged well into the following day.

At 6 p.m. on May 22 there was a bluish discoloration of the skin in the left antecubital fossa irregularly distributed around the superficial ulnar artery, the patch having a crenated border and being surrounded by an area of pallor. The limb otherwise was warm and pink, with palpable radial and ulnar pulses. Oral tolazoline, $25 \mathrm{mg}$. six-hourly, was begun, the limb being kept under the closest observation. Subsequently the patch began to fade after three days, and the patient was finally discharged from hospital on the thirteenth day after operation. He was seen again as an out-patient 10 days later, when the condition of the limb was satisfactory.

\section{Discussion}

The incidence of accidental intra-arterial injection of thiopentone has been variously given as 3 in 25,000 (Lundy, 1942) and 1 in 55,000 (Cohen, 1948).

When such a mishap occurs it is essential to appreciate the significance of the catastrophe. Of 12 reported cases, 6 required arm or forearm amputation, 2 suffered loss of one or more fingers, and 2 suffered severe residual disability from nerve effects. The immediate sequel to this mishap is an intense arterial spasm, especially of the distal arteriolar tree, with possible trapping or delay of some of the solution. Such spasm may persist for at least 24 hours, and is very easily precipitated when injection has been made into a superficial ulnar artery, as these vessels are derived from the vasa aberrantia, and, like other superficial vessels, have a fuller nerve supply than the deeper branches. Thrombosis is the essential pathological lesion, although it is not yet clear why the arterial tree should show such a vigorous reaction when venous thrombosis is relatively uncommon. Thiopentone is a strongly alkaline irritant, and the intima of the artery appears to be particularly sensitive to solutions of such high $\mathrm{pH} ;$ a further factor may be the resistance of the arterial wall due to the relative abundance of muscle and elastic fibres. The injection of $10 \%$ solution is of much graver prognostic significance than either the $5 \%$ or $2 \frac{1}{2} \%$. In eight of the published cases, gangrene occurred in four, and in three of these a $10 \%$ solution had been employed.

The ease with which the catastrophe may occur has been aptly described by Cohen, and his words are particularly applicable to this case when he states: "There may be little groping for the vein and the injection has often been particularly easy. Intra-arterial puncture is not followed, as is commonly imagined, by a gush of blood blowing off the syringe piston. Nor is the crimson livery of arterial blood readily discerned in the yellowish solution."

The use of a safety pause after injection of a certain amount of solution should be obligatory. It would appear, however, that the test injection of small amounts up to $1 \mathrm{ml}$. of $5 \%$ solution, as commonly recommended, is not wholly reliable, and it is suggested that $2 \mathrm{ml}$. would provide a more reliable test injection.

The pain produced on intra-arterial injection is agonizing and of a characteristic burning nature well described as a sensation of the hand being on fire. In the present case the patient on leaving hospital 14 days later could still vividly recall the burning nature of the pain in his left forearm and hand. This pain, together with the very obvious delay in the onset of narcosis, should make recognition of the accident a certainty and convince even the most obtuse of the mishap.

Once the accident has occurred the operation should be abandoned if at all possible, as the subsequent heparinization, with risk of haemorrhage from the operation site, otherwise proves a constant source of anxiety.

The presence of palpable radial and ulnar pulses does not exclude the possibility of severe damage to the intima and 
subsequent thrombosis. The pulse may disappear after a few hours, or it may remain full until shortly before the onset of gangrene, as shown in three published cases, where gangrene ensued after disappearance of the pulse on the eighth, tenth, and sixteenth days, respectively. In these circumstances the only answer to this condition, once it has occurred, is intensive treatment, the necessity for which remains undiminished by the presence of palpable pulses in the affected limb.

The appearance of discoloured skin patches in the affected limb has been described in several of the previously reported cases. They have also been reported as a complication of arteriography and ascribed by Wagner (1944) to secondary spread of thrombosis from the main vessel to distal cutaneous branches. In the present case, however, with no brachial artery thrombosis, the patch would seem to have been due to a direct local effect of the thiopentone shunted into the skin vessels.

The treatment of this condition is directed towards ensuring full vasodilatation of the limb with obliteration of all arterial spasm, the prevention of thrombosis, and satisfactory venous drainage. There is no place for complacency: once the mistake has been recognized, treatment must be started immediately and followed through intensively. The experience gained from the present case emphasizes the fact that, if at all possible, the operation should not be allowed to continue, as was permitted in this instance and subsequently regretted when oozing from the penis became an additional source of worry on the second and third days.

As the patient is unconscious when the first brachial plexus block is performed immediately after the accident, and consequently paraesthesiae or other corroboration of the success of the block cannot be obtained, it is advised that Patrick's method be employed, using at least $20 \mathrm{ml}$. of solution, preferably with hyalase. The repetition of the block in the present case, several hours later after heparinization had begun, together with the intra-arterial injection of tolazoline and heparin, was recognized as a hazardous procedure, but it was felt that the vascular supply to the limb at this stage was jeopardized to a precarious extent by the arterial spasm.

Tolazoline has been the subject of a report on peripheral vascular disease by Edwards et al. (1952). These workers found it to be one of the best vasodilators available, and recommended a dosage of 50-100 mg. at weekly intervals. In addition, they state that "Swan's plethysmographic studies (quoted by Lynn, 1950) demonstrated an increased blood flow in an already sympathectomized limb following the intra-arterial injection of $50 \mathrm{mg}$. of tolazoline." This drug would thus appear to have properties of much value in the treatment of cases of intra-arterial thiopentone, not least of which is its prolonged action after intra-arterial injection. It is of interest to note that the same workers showed that intra-arterial procaine had little or no value in producing an increase in peripheral limb blood flow. It is suggested, therefore, that immediate treatment for cases of accidental intra-arterial thiopentone should include intra-arterial tolazoline, in addition to the brachial plexus block, a procedure easily accomplished, as Dundee (1953) states, by puncturing the subclavian artery on completion of the block. In the case under review tolazoline was not immediately available, but when it was injected into the subclavian artery the vasodilator response was both dramatic and prolonged.

Other methods of assisting vasodilatation and forcing the collateral circulation should be employed, especially warmth and sedation. The use of morphine has been shown by Learmonth (1946) to relax vessel spasm, although in the present case vomiting became a troublesome complication, which ceased on the substitution of pethidine.

In view of reports of delayed onset of gangrene in several cases, it is difficult to say for how long these measures should be maintained. In the present case the strict regime was not relaxed until the sixth day, and the patient remained under observation until the thirteenth day. By this time the danger from arterial spasm is well past, and, in the absence of any significant sign of impaired limb blood flow, it is difficult to conceive the danger of delayed thrombosis being anything but remote.

\section{Summary}

A case of accidental intra-arterial injection of thiopentone is reported with a description of the treatment employed.

A test injection of $2 \mathrm{ml}$. with a safety pause is advocated as a preventive measure.

The operation should be abandoned, if at all possible, once the mishap has occurred.

The necessity for immediate and intensive treatment is stressed.

The use of intra-arterial tolazoline is recommended.

I wish to thank Dr. A. C. Forrester for permission to publish this case, and to express my appreciation to Professor W. A. Mackey for his advice.

REFERENCES

Cohen, S. M. (1948). Lancet, 2, 361, 409.

Dundee, J. W. (1953). British Medical Journal, 1, 402

Edwards, J. W. L., Jones, N. B.. McConnell, R. B., Pemberton, H. S. and Watson, D. C. (1952). Ibid. 2, 808,

Learmonth, J. R. (1946). Proc. roy. Soc. Med, 39, 488

Lundy, J. S. (1942). Clinical Anesthesia, p. 542. Saunders, London.

Lynn. R. B. (1950). Lancet, 2, 676.

Wagner, F. B. (1944). J. Amer. med. Ass., 125, 958.

\section{TOTAL PAROTIDECTOMY WITH PRESERVATION OF FACIAL NERVE}

\section{A REVIEW OF 47 CASES}

BY

\section{H. A. KIDD, F.R.C.S.Ed., M.R.C.O.G.}

Consultant Surgeon, St. Helier Hospital, Carshalton, Surrey

The operation of total parotidectomy with preservation of the facial nerve for non-malignant conditions as advocated by Hamilton Bailey (1941) is slowly gaining adherents in this country, and 47 patients who have had this operation have now been followed up to try to ascertain the answers to three questions: (1) Does total removal of the parotid gland reduce the number of recurrences which follow local removal of mixed parotid tumours? (2) Can total removal of the parotid gland be performed and full function of the facial nerve be preserved in every case? (3) What is the cause of the Frei syndrome and how may it be prevented ?

The technique of the operation used in these cases has been described elsewhere (Kidd, 1950, 1953), and the value of starting the dissection by locating the branch of the cervico-facial nerve going to the lower lip is confirmed. Increased familiarity with the operation favours the preservation of the branch of the great auricular nerve going to the lobe of the ear and, in many cases, deliberate resection of the auriculo-temporal nerve.

\section{Diagnosis}

It is not perhaps generally recognized how many different primarily non-malignant conditions arise in the parotid gland, nor how difficult it can be to be sure that a tumour in the parotid area is actually in the gland. Many true parotid tumours feel very superficial and mobile, and deepseated tumours may be found to arise from deep lymphatic glands. The history and careful palpation are the best guides; good sialograms are also of value, but in some cases only exploration will clinch the diagnosis. As the danger of cutting into a mixed parotid tumour is very real it is wise to plan the incision and approach, in a difficult 